\title{
Impact of ultrasonography detected quadriceps calcific tendinopathy on pain and function in patients with primary knee osteoarthritis
}

\author{
M.A. Mortada, L.I. Kotb, Y.A. Amer \\ Rheumatology and Rehabilitation Department, Faculty of Medicine, Zagazig University, Egypt
}

\begin{abstract}
Calcific tendinopathy is most commonly seen around the shoulder joint. Only a few cases of quadriceps calcific tendinopathy (QCT) were reported. This study compares pain, function, clinical examination results, and ultrasonographic findings among primary knee osteoarthritis (KOA) patients with or without ultrasonographydetected QCT.

A cross-sectional study was conducted on 214 patients with knee OA. Ultrasonography (US) of knee joints was performed according to the EULAR guidelines. Kellgren-Lawrence radiographic grading was used to score OA. Pain and functional status were assessed using the visual analog scale (VAS), the Health Assessment Questionnaire-II (HAQ-II), and the Western Ontario and McMaster Universities Osteoarthritis Index (WOMAC). QCT was detected in 50 out of 428 knees (11.6\%), i.e. in 46 out of 214 patients (21.49\%). Most cases of QCT were detected in the following sites: 36 in the vastus lateralis (72\%), 10 in the vastus intermedius (20\%), and only 4 in the vastus medialis (8\%). QCT was found mainly in advanced KOA stages: 44 cases of QCT were found in patients with grade $4 \mathrm{KOA}$ and 6 cases in grade $3 \mathrm{KOA}$. The presence of QCT showed a statistically significant association $(p<0.05)$ with VAS, HAQ-II, WOMAC subscales, synovitis, and effusion detected by US. In knees with ultrasound-detected QCT, ultrasonographic features of CPPD were found in 31 knees (62\%). QCT was found in cases with advanced KOA and mainly with ultrasonographic findings of CPPD disease. QCT could be considered an independent poor prognostic finding regarding pain, functional activity, and response to NSAIDs.
\end{abstract}

Key words: Quadriceps, calcific tendinopathy, ultrasonography, CPPD, knee.

Reumatismo, 2021; 73 (2): 111-116

\section{INTRODUCTION}

alcific tendinopathy is seen in $3-15 \%$ of the general population (1-3). It is thought to be a form of dystrophic calcification resulting from repeated trauma. However, the exact pathophysiology remains unclear (4). Calcific tendinopathy affects most commonly the shoulder (the supraspinatus tendon), followed by wrist, hip and elbow. However, any tendon can be affected (5). Only a few studies assessed quadriceps calcific tendinopathy (QCT) by radiography, with a reported frequency of about $8.4 \%$ (4, 6-8).

Ultrasound (US) is known to be more sensitive than radiographs in depicting milder degrees of calcification (9). The clinical and functional consequences of QCT are still largely unknown.

The objective of this study is to compare pain, function, and clinical and ultrasonographic findings among primary KOA patients with or without US-detected QCT.

\section{MATERIALS AND METHODS}

\section{Study design}

A single-center cross-sectional study was conducted in the outpatient clinics of the Rheumatology and Rehabilitation Department, Zagazig University Hospitals (Egypt), from February 2019 to July 2019. The study was approved by the Institutional Review Board at the Faculty of Medicine, Zagazig University Hospitals,
Corresponding author: Mohamed Mortada

Rheumatology and Rehabilitation Department Faculty of Medicine, Zagazig University Al-Qawmia Street, P.O. Box: 44519 Zagazig, Sharqia Governorate, Egypt E-mail: m_a_mortada@yahoo.com 
and the Rheumatology and Rehabilitation Department at the same University. The study was performed according to the Code of Ethics of the World Medical Association (Declaration of Helsinki 1964) for studies involving humans. Written informed consent was obtained from each participant.

\section{Patients}

This study included 214 consecutive patients (34 men and 180 women) aged 45 and older, who fulfilled the American College of Rheumatology clinical classification criteria for KOA (10).

The exclusion criteria were: history of any knee surgeries; secondary KOA to other forms of arthritis such as rheumatoid arthritis or infectious arthritis; history of malignancy or trauma; a current stroke or neurological diseases.

\section{Clinical assessment}

A full history and clinical locomotor examinations were performed on all patients. The height and weight of each patient were recorded for body mass index (BMI) calculation.

After the exclusion criteria were applied, the following parameters were measured to assess the knee pain severity and the functional capability:

1) VAS as a subjective measure for evaluating pain intensity. Patients indicated their degree of pain on a scale of 0 (no pain) to 10 (extreme pain);

2) The Western Ontario and McMaster Universities Osteoarthritis Index (WOMAC) was calculated based on the Likert version. The index is divided into three subscales: pain (five items), stiffness (two items), and physical function (17 items). The minimum score is 0 and the maximum score is 96 ( 20 points for pain, 8 points for joint stiffness, and 68 points for physical function) (11);

3) HAQ-II (Health Assessment Questionnaire-II) as a reliable and valid ten-item functional questionnaire used over the same week of the examination, which correlates more with clinical variables than other HAQ scales (12).
All patients were asked about their previous responses to NSAIDs and analgesics other than tramadol.

\section{Radiographic assessment}

Routine weight-bearing anteroposterior and lateral knee radiographs were taken and analyzed by a rheumatologist blinded to the clinical and musculoskeletal ultrasonography (MSUS) findings. Severity was assessed, and the patients were graded from zero to four according to KellgrenLawrence Grading (KLG) (13). Radiographic diagnosis of QCT appeared as whiskers or flecks where calcification had its origin from the anterior superior edge of the patella and fanned out into the quadriceps tendon. This calcific whisker was radiologically distinct from osteophytic spurs that occur at the posterior margin of the patella (6).

\section{Musculoskeletal ultrasound examination}

Musculoskeletal ultrasound (MSUS) evaluations were conducted on both knees (428 knees) according to the EULAR guidelines (14) by a rheumatologist, who was experienced in conducting MSUS and blinded to the clinical and radiographic evaluations. All patients were examined using a B-mode (grayscale) real-time MSUS (LOGIQE10 GE, Healthcare) interfaced with a $10-18 \mathrm{MHz}$ linear array transducer. The anterior aspect of the knee was examined with the patient in the supine position. A knee flexion of approximately $20^{\circ}-30^{\circ}$ obtained by placing a small pillow beneath the popliteal space stretched the extensor mechanism and avoided possible anisotropy related to the concave profile that the quadriceps and patellar tendons take in full extension. Sagittal US images obtained in the midline, while keeping the distal edge of the probe over the patella, display the quadriceps tendon. On the long-axis and short-axis planes, the multilayered appearance and the quadriceps tendon components (rectus femoris; vastus medialis and lateralis; vastus intermedius) could be observed.

Quadriceps calcific tendinopathy (QCT) was defined as a hyperechoic mass found 


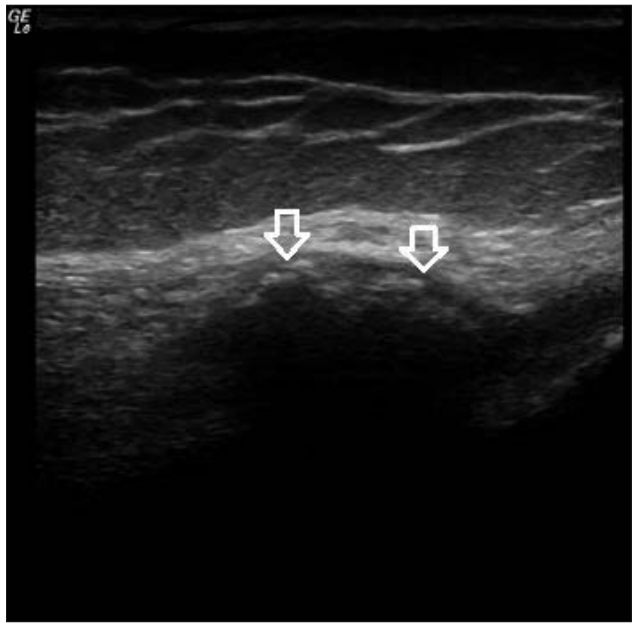

Figure 1 - Longitudinal (long) scan of the anterior knee at the suprapatellar pouch with quadriceps calcific tendinopathy, which appears as a hyperechoic mass within the vastus lateralis tendon with posterior shadowing.

within the quadriceps tendon with posterior shadowing (Figures 1 and 2). All cases of US-detected QCT had an additional confirmatory US examination by another rheumatologist experienced in MSUS. In cases of inconsistency, a consensus on US diagnosis of QCT was reached.

US features of CPPD in knees were detected according to the OMERACT guidelines (15) in the form of hyperechoic deposits of variable shape localized within the meniscus (medial/lateral) or femoral hyaline cartilage. Both types of deposits remained fixed and moved during the dynamic assessment (flexion and extension of knee).

\section{Statistical analysis}

The collected data were coded, entered, presented, and analyzed by a computer using a database software program, Statistical Package for Social Science (SPSS) (Version 20.0. Armonk, NY: IBM Corp). Quantitative variables were expressed as the mean \pm standard deviation (SD), whereas the qualitative variables were expressed as numbers and percentages. For quantitative variables, independent samples $t$-test $(t)$ for normally distributed data was used, while nonparametric data were

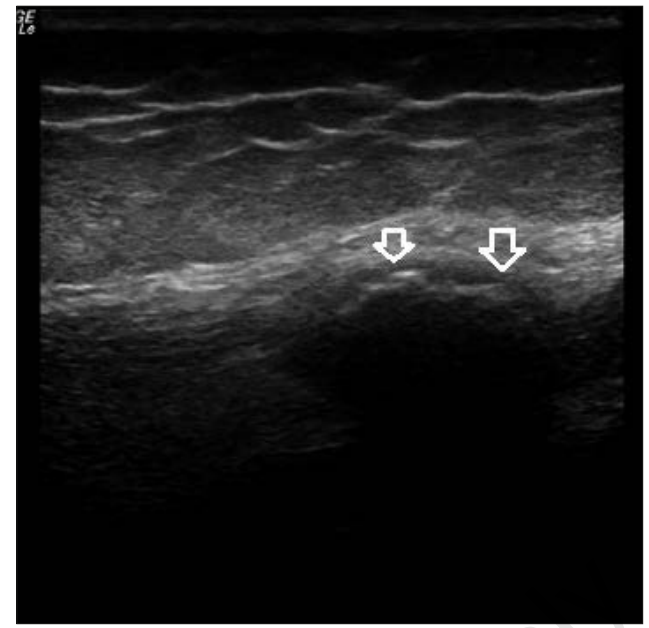

Figure 2 - Transverse scan of the anterior knee at the suprapatellar pouch with quadriceps calcific tendinopathy, which appears as a hyperechoic mass within the vastus lateralis tendon with posterior shadowing.

evaluated with the Mann-Whitney $U$ Test. The chi-square test was used to detect the relationship between different qualitative variables. The results were considered statistically significant and highly statistically significant when the significant probability value ( $p$ value) was $<0.05$ and $<0.001$, respectively.

\section{RESULTS}

Demographic data for the studied group of patients with KOA are shown in Table I.

Using US, QCT was detected in 50 knees (11.6\% of examined knees) of 46 out of 214 patients. Bilateral QCT was detected only in four patients. Most cases of QCT were found in the vastus lateralis in 36 knees (72\% of QCT cases), in the vastus intermedius in ten knees (20\% of QCT cases), and in the vastus medialis in only four cases (Table II).

Ultrasonographic-detected QCT was confirmed by radiography in 35 cases $(70 \%$ of QCT cases), representing $8.2 \%$ of all cases. All $(100 \%)$ radiography-detected cases were detected by US.

QCT was present mainly in the advanced stages of KOA: 44 cases of QCT were found in patients with grade $4 \mathrm{KOA}$ and six 
Table I - Demographic and clinical characteristics of patients with and without quadriceps calcific tendinopathy.

\begin{tabular}{|c|c|c|c|c|}
\hline Variables & $\begin{array}{c}\text { Total } \\
\text { (No. =214) }\end{array}$ & $\begin{array}{l}\text { Patients without QCT } \\
(\text { No. }=165)\end{array}$ & $\begin{array}{c}\text { Patients with QCT } \\
\text { (No.=49) }\end{array}$ & $p$ value \\
\hline Women, total 180 (No.) & 180 & 133 & 47 & \\
\hline Age, years (mean $\pm S D)$ & $57.4( \pm 8.2)$ & $57.1 \pm 8.9$ & $56.3 \pm 7.8$ & 0.57 \\
\hline Disease duration, years (mean $\pm S D$ ) & $16.3( \pm 5.2)$ & $15.1 \pm 4.2$ & $17.9 \pm 5.1$ & 0.001 \\
\hline Body mass index, kg/m2 (mean \pm SD) & $34.4( \pm 4.2)$ & $33.1 \pm 4.8$ & $35.9 \pm 4.9$ & 0.0004 \\
\hline WOMAC pain subscale (mean $\pm S D$ ) & $13.2( \pm 4.2)$ & $10.3 \pm 2.1$ & $16.2 \pm 5.2$ & 0.0001 \\
\hline WOMAC stiffness subscale (mean \pm SD) & $5.5( \pm 0.5)$ & $4.5 \pm 1.1$ & $6.1 \pm 1.2$ & 0.0001 \\
\hline WOMAC function subscale (mean \pm SD) & $39.5( \pm 8.1)$ & $25.5 \pm 6.1$ & $45.3 \pm 10.1$ & 0.0001 \\
\hline WOMAC total scale $($ mean $\pm S D)$ & $58.3( \pm 10.1)$ & $60.1 \pm 10.5$ & $68.1 \pm 8.9$ & 0.0001 \\
\hline VAS $($ mean \pm SD) & $74.0( \pm 14.6)$ & $59.4 \pm 11.5$ & $77.1 \pm 17.1$ & 0.0001 \\
\hline $\mathrm{HAQ}-\mathrm{II}($ mean $\pm \mathrm{SD})$ & $6.7( \pm 3.2)$ & $5.4 \pm 1.6$ & $8.2 \pm 3.2$ & 0.0001 \\
\hline
\end{tabular}

No., number; SD, standard deviation; OA, osteoarthritis; WOMAC, Western Ontario and McMaster Universities Index; VAS, visual analog scale. ${ }^{*} p$ value $<0.001$ is highly significant.

Table II - The comparison between patients with and without ultrasonography-detected quadriceps calcific tendinopathy.

\begin{tabular}{|c|c|c|c|c|}
\hline \multirow{2}{*}{\multicolumn{2}{|c|}{ Variables }} & \multicolumn{2}{|c|}{ Ultrasonographic finding of QCT } & \multirow[b]{2}{*}{$p$ value } \\
\hline & & $\begin{array}{c}\text { Yes } \\
\text { No. of knees }=50\end{array}$ & $\begin{array}{c}\text { No } \\
\text { No. of knees }=378\end{array}$ & \\
\hline \multicolumn{2}{|c|}{$\begin{array}{l}\text { Osteoarthritis grade ( } n=428 \text { knees) } \\
\text { I }(n=53) \\
\text { II }(n=177) \\
\text { III }(n=102) \\
\text { IV }(n=96)\end{array}$} & $\begin{array}{c}0(0 \%) \\
0(0 \%) \\
6(12 \%) \\
44(88 \%)\end{array}$ & $\begin{array}{c}53(14 \%) \\
177(46.8 \%) \\
96(25.4 \%) \\
52(13.8 \%)\end{array}$ & 0.001 \\
\hline \multicolumn{2}{|c|}{ Radiography finding QCT ( $n=428$ knees) } & \multirow[b]{2}{*}{$\begin{array}{l}35(70 \%) \\
15(30 \%)\end{array}$} & \multirow[b]{2}{*}{$\begin{array}{c}0(0 \%) \\
378(100 \%)\end{array}$} & \multirow[b]{2}{*}{0.001} \\
\hline $\begin{array}{l}\text { Yes } \\
\text { No }\end{array}$ & $\begin{array}{l}35(8.2 \%) \\
393(91.8 \%)\end{array}$ & & & \\
\hline \multicolumn{2}{|c|}{ Ultrasonographic finding of CPPD ( $n=428$ knees) } & \multirow[b]{2}{*}{$\begin{array}{l}31(62 \%) \\
19(38 \%)\end{array}$} & \multirow[b]{2}{*}{$\begin{array}{c}47(12.4 \%) \\
331(87.6 \%)\end{array}$} & \multirow[b]{2}{*}{0.02} \\
\hline $\begin{array}{l}\text { Yes } \\
\text { No }\end{array}$ & $\begin{array}{l}78(18.2 \%) \\
350(81.2 \%) \\
\end{array}$ & & & \\
\hline \multicolumn{2}{|c|}{$\begin{array}{l}\text { Poor response to NSAIDs and analgesics } \\
\text { ( } n=214 \text { cases) }\end{array}$} & $40(80 \%)$ & $79(20.9 \%)$ & 0.001 \\
\hline
\end{tabular}

${ }^{*} p$ value $<0.05$ is significant; ** $p$ value $<0.001$ is highly significant.

cases in grade $3 \mathrm{KOA}$. Other features detected by US included the following: effusions in 385 knees $(89.9 \%)$, synovial swelling in 243 knees $(56.8 \%)$, and Baker's cyst in 376 knees $(87.9 \%)$.

The presence of ultrasonographic-detected QCT showed a statistically significant association $(p<0.05)$ with age, VAS, HAQII, WOMAC subscales, synovitis, and effusion. A poor response to NSAIDs and analgesics was reported by 40 patients (86.9\%) with US-detected QCT and 79 patients $(45.9 \%)$ without US-detected QCT $(\mathrm{p}=0.001)$. Patients with grade $4 \mathrm{KOA}$ and
US-detected QCT had significantly higher values of VAS, HAQ-II, WOMAC subscales, synovitis, and effusion.

Ultrasonographic features of CPPD were detected in 78 knees (18.2\%), mainly in grade $4 \mathrm{KOA}$ (32 knees $33.3 \%$ ). In knees with US-detected QCT, US features of CPPD were found in 31 knees (62\%).

\section{DISCUSSION}

Quadriceps tendon calcification was previously reported in few case reports and radiological studies $(4,6-8)$. 
This is the first study to detect the impact of US-detected QCT on KOA to the best of our knowledge. In this study, we detected more QCT by US (11.6\%) than by radiography $(8.2 \%)$ in KOA cases probably due to the high sensitivity of MSUS in the detection of calcifications (11). The reported frequency in our study is consistent with the results of Yang et al., who reviewed 225 lateral knee radiographs from 138 patients to document quadriceps and gastrocnemius tendon calcification. They found gastrocnemius tendon calcification in $28.4 \%$ of knees, which was significantly more common than quadriceps tendon calcification $(8.4 \%)(16)$.

The US-detected QCT was found in advanced KOA cases, and most of our cases were associated with US features of CPPD disease. These results may indicate that US-detected QCT may be a part of CPPD disease, which tends to present in advanced KOA stages. Similarly, all radiographically detected QCT cases reported by Yang et al. were associated with evidence of chondrocalcinosis (16).

More importantly, patients with US-detected QCT had significantly more pain, higher scores of total \& subtotals WOMAC scale, functional impairment, and poor response to NSAIDs, consistent with Mohammed et al. (17), who found that the mean WOMAC pain score was significantly higher in patients with crystal deposits.

This result could not be explained totally by the association of US-detected QCT with advanced KOA stages, which occurred in patients with grade 4. Patients with US-detected QCT still had a more significant clinical and functional impairment. Subsequently, US-detected QCT could be considered as an independent poor prognostic finding.

However, this study has some limitations. Firstly, we did not perform a pathological analysis of the detected calcification to validate its nature as hydroxyapatite crystals. Secondly, we did not assess other conditions that may predispose to calcific tendinopathy, specifically, dysmetabolism, hyperuricemia, and physical activity.

\section{CONCLUSIONS}

In conclusion, US can detect QCT more efficiently than radiography in patients with KOA. QCT is found in advanced KOA cases and, mainly, with other ultrasonographic findings of CPPD disease. QCT could be considered an independent poor prognostic finding in terms of pain, functional activity, and response to NSAIDs.

\section{Authors' contributions}

Each author made significant individual contributions to this manuscript. Each author believes that the manuscript represents truthful work. All authors approved the entirety of the submitted material and contributed actively to the study.

\section{Ethical statement}

Written informed consent was obtained from each participant. The study was approved by the ethical committee of the institute and the study was conducted in compliance with the Declaration of Helsinki.

\section{Acknowledgements}

None declared.

\section{REFERENCES}

1. Speed CA, Hazleman BL. Calcific tendinitis of the shoulder. N Engl J Med. 1999; 340: 1582-1584.

2. Bosworth BW. Calcium deposits in the shoulder and subacromial bursitis. A survey of 12, 222 shoulders. JAMA. 1941a; 116: 24772482.

3. Bosworth BW. Examination of the shoulder for calcium deposits. J Bone Joint Surg Am. 1941b; 23: 567-577.

4. Holt PD, Keats TE. Calcific tendinitis: a review of the usual and unusual. Skeletal Radiol. 1993; 22: 1-9.

5. Pereira BP, Chang EY, Resnick DL, Pathria MN. Intramuscular migration of calcium hydroxyapatite crystal deposits involving the rotator cuff tendons of the shoulder: report of 11 patients. Skeletal Radiol. 2016; 45: 97-103.

6. Trujeque L. Patellar whiskers and acute calcific quadriceps tendonitis in a general hospital population. Arthritis Rheum. 1977; 20: 1409-1412.

7. Macurak RB, Goldman J A, Hirsh E, et al. Acute calcific quadriceps tendonitis. South Med J. 1980; 73: 322-325. 
8. Bhole R, Flynn JC, Marbury TC. Quadriceps tendon ruptures in uremia. Clin Orthop Relat Res.1985; 195: 200-206.

9. Freire V, Moser TP, Lepage-Saucier M. Radiological identification and analysis of soft tissue musculoskeletal calcifications. Insights Imaging. 2018; 9: 477-492.

10. Altman R, Asch E, Bloch D, et al. Development of criteria for the classification and reporting of osteoarthritis. Classification of osteoarthritis of the knee. Diagnostic and Therapeutic Criteria Committee of the American Rheumatism Association. Arthritis Rheum. 1986; 29: 1039-1049.

11. Bellamy N, Buchanan WW, Goldsmith $\mathrm{CH}$, et al. Validation study of WOMAC: a health status instrument for measuring clinically important patient relevant outcomes to antirheumatic drug therapy in patients with osteoarthritis of the hip or knee. J Rheumatol. 1988; 1833-1840.

12. Wolfe F, Michaud K, Pincus T. Development and validation of the health assessment questionnaire II: a revised version of the health assessment questionnaire. Arthritis Rheum. 2004; 50: 3296-3305.
13. Kellgren JH, Lawrence JS. Radiological assessment of osteo-arthrosis. Ann Rheum Dis. 1957; 16: 494-502.

14. Möller I, Janta I, Backhaus M, et al. The 2017 EULAR standardised procedures for ultrasound imaging in rheumatology. Ann Rheum Dis. 2017; 76: 1974-1979.

15. Filippou G, Scirè CA, Damjanov N, et al. Definition and reliability assessment of elementary ultrasonographic findings in calcium pyrophosphate deposition disease: a study by the OMERACT calcium pyrophosphate deposition disease ultrasound subtask force. $\mathrm{J}$ Rheumatol. 2017; 44: 1744-1749.

16. Yang BY, Sartoris DJ, Resnick D, Clopton P. Calcium pyrophosphate dihydrate crystal deposition disease: frequency of tendon calcification about the knee. J Rheumatol. 1996; 23 : 883-888.

17. Mohammed R, Kotb H, Amir M, Di Matteo A. Subclinical crystal arthropathy: a silent contributor to inflammation and functional disability in knees with osteoarthritis - an ultrasound study. J Med Ultrasonics. 2019; 46: 137-146. 